\title{
Piezosurgery in Bone Augmentation Procedures Previous to Dental Implant Surgery: A Review of the Literature
}

\author{
Gabriel Leonardo Magrin, Eder Alberto Sigua-Rodriguez ${ }^{*}$, Douglas Rangel Goulart and \\ Luciana Asprino
}

Piracicaba Dental School, State University of Campinas, Piracicaba, Brazil

\begin{abstract}
The piezosurgery has been used with increasing frequency and applicability by health professionals, especially those who deal with dental implants. The concept of piezoelectricity has emerged in the nineteenth century, but it was applied in oral surgery from 1988 by Tomaso Vercellotti. It consists of an ultrasonic device able to cut mineralized bone tissue, without injuring the adjacent soft tissue. It also has several advantages when compared to conventional techniques with drills and saws, such as the production of a precise, clean and low bleed bone cut that shows positive biological results. In dental implants surgery, it has been used for maxillary sinus lifting, removal of bone blocks, distraction osteogenesis, lateralization of the inferior alveolar nerve, split crest of alveolar ridge and even for dental implants placement. The purpose of this paper is to discuss the use of piezosurgery in bone augmentation procedures used previously to dental implants placement.
\end{abstract}

Keywords: Dental implants, jaw, oral surgery, osteotomy, piezosurgery, sinus floor augmentation.

\section{INTRODUCTION}

There are several challenges faced by Oral Surgeons engaged in dental implantology. To overcome these challenges and make them less arduous, a lot of techniques, devices and gadgets are developed and tested by the scientific community. Among those devices, the piezoelectric surgical ultrasonic device has emerged as a real possibility of improvement in the performance of medical and dental surgical procedures.

In dentistry, the piezoelectric bone surgery was developed by the oral surgeon Tomaso Vercellotti in 1988, to overcome the limitations of traditional instrumentation in oral bone surgery [1]. Piezoelectric osteotomy devices are based on the modulation of ultrasonic vibration of an active tip called insert and are characterized by three essential points: precise and clean cutting, selective bone-cutting and surgical field relatively free of blood [1]. As a result of those characteristics, piezoelectric osteotomies provide a cut in bone structure maintaining the integrity of the surrounding soft tissues [2]. In addition, the piezoelectric device is easy to handle and can be learned quickly [3].

Piezoelectricity is a physical phenomenon in which an electrical charge is formed on the surface of certain crystals, such as quartz, when they are subjected to compressive forces [4]. In piezosurgery (PS), this physical principle is used in "reverse order". An alternating electrical current in average frequency is transmitted to the crystals, causing their expansion and retraction repeatedly, and producing mechanical

*Address correspondence to this author at the Piracicaba Dental School, Department of Oral Diagnosis. P.O. Box 52, State University of Campinas UNICAMP. Piracicaba, São Paulo - Brazil; Tel: (55) 19- 2106 5325;

E-mail: edersiguaodont@gmail.com oscillations. These oscillations generate ultrasonic waves that are sent to the tip of the piezoelectric hand piece and, when used in short and fast movements, are able to disrupt and fragment solid segments such as bone tissue [5]. Soft tissues will be affected and could be cut by the ultrasonic vibration in frequencies over $50 \mathrm{kHz}$ [6]. Once the frequency used for bone cutting (between 25 and $30 \mathrm{kHz}$ ) only affects hard tissues, soft tissues are preserved and can be touched by the tip of the instrument without any harm, turning the piezoelectric device unique in surgery.

Due to the advantageous aspects of PS uses in comparison with traditional techniques, the number of indications for the device has been increasing in oral and maxillofacial surgery and in other areas as otorhinolaryngology, neurosurgery, ophthalmology, orthopedics and traumatology [7, 8]. And also, the literature presents a lot of case reports and surveys with the application of the piezoelectric device. The purpose of this paper is to discuss the use of PS in bone augmentation procedures used previously to dental implants placement.

\section{LITERATURE REVIEW}

Piezosurgery is based on piezoelectric effect which states that certain ceramics and crystals deform when an electric current passes through them, resulting in oscillations of ultrasonic frequency [6]. The vibrations obtained are amplified and transferred to a vibration tip, which when applied with light pressure on bone tissue results in a cavitation phenomenon, an effect of mechanical cutting which occurs exclusively in mineralized tissues [9].

Conventional techniques with drills and cutting discs have been showing some disadvantages when compared with the piezoelectric osteotomy, such as overheating and possible damages to adjacent tissues [10]. In the osteotomy pro- 
cedures the use of this technique showed best biological results in bone remodeling process when it was compared to conventional techniques with saws and drills [11].

Vercellotti et al. (2005) analyzed bone remodeling process after piezoelectric osteotomy compared with the conventional techniques which used carbide and diamond burs. It was possible to conclude that PS provided more favorable bone repair when osteotomy and osteoplasty were performed. Therefore, PS could be considered efficient in bone surgery [12].

In contrast to conventional microsaws where blood is moved in and out of the cutting area and the visibility is low, the operative field in PS remains almost blood-free during cutting procedure [13]. Other authors have demonstrated a reduction in inflammatory cells and increased osteogenic activity around implants placed by piezoelectric ultrasound device in comparison with conventional drills systems $[14,15]$.

Piezosurgery produces less vibration and noise than conventional surgery with burs and saws because it uses microvibrations. These features could minimize patient's psychological stress and fear while the osteotomy is performed under local anesthesia [16]. Furthermore, the device is useful in cases of cutting bone adjacent to important soft tissues structures such as nerves, vessels, Schneiderian membrane and duramater, where mechanical and thermal injuries must be avoided [17].

\section{Use for Sinus Lifting Surgery}

The first indication of the piezoelectric surgery device in dental implantology was maxillary sinus lifting surgery [18]. Dental implants placement in the posterior maxilla often finds the maxillary sinus as an anatomical structure which offers a limit to the procedure. Superior premolars and molars edentulism is associated with atrophy of the maxilla and sinus pneumatization that hampered implant placement in this area. The maxillary sinus floor augmentation has been used successfully in the treatment of these cases $[19,20]$.

The most widely used technique for lifting the sinus floor is the classic sidewall antrostomy, introduced by Tatum in 1976 and subsequently described by Boyne in 1980 [21]. Another famous technique use osteotomes in the alveolar crest, proposed by Summers in 1994 [22]. The decision about which surgical technique perform depends on the remaining bone between the alveolar crest and the floor of the maxillary sinus.

The hydrodynamic pressure applied by irrigating solution in PS helps in sinus membrane dissection. Using piezoelectric ultrasonic vibration of $25-30 \mathrm{kHz}$, the device cuts only mineralized structures without cutting the soft tissue. The cavitation effect of the system induces a hydropneumatic pressure of saline irrigant that contributes to the atraumatic elevation of the sinus membrane [1]. When the osteotomy of the sidewall of maxillary sinus is performed with piezoelectric ultrasonic vibration the bone loss is usually lower [23].

Vercellotti et al. (2001) reported that inadvertent perforation of the membrane is unlikely when piezosurgery technique is applied appropriately [18]. Flemming et al., in 1998, illustrated this method in a study with 15 patients in which
21 piezoelectric osteotomies were performed. They found a success rate of $95 \%$. Perforations in the maxillary sinus membrane were observed in only $5 \%$ of patients [24]. Wallace et al. (2007) conducted a study in which 100 maxillary sinus surgeries were performed using the piezoelectric device. Only 7 cases of perforation of the sinus mucosa were observed. None of these perforations occurred because of the inserts of the piezoelectric unit. All of them were caused by subsequent elevation of the Schneiderian membrane with hand tools. Perforations occurred due to the presence of bony septum ( 4 cases) and by manipulation of extremely thin membranes (3 cases) [19].

Another advantage of piezoelectric ultrasonic vibration is its accuracy. Compared with micro-oscillating saw, the movement of the active tip of the piezosurgical device is small. Therefore, the cutting accuracy is increased and causes less discomfort to the patient [13]. All lateral antrostomy can be performed with the piezoelectric device inserts. The bone removed by osteotomy may be collected and can be incorporated into the graft that will fill the sinus. The Schneiderian membrane can be detached from the bone with a cone compressor tip without any damage [7].

\section{Use for Bone Graft Surgery}

Autogenous bone graft particles of 500 micrometers are ideal for bone regeneration, once they maintain the osteogenic, osteoinductive and osteoconductive potentials [25]. Bone graft surgery is commonly carried out through osteotomies in intraoral bone donor sites, with the aid of surgical drills and saws [26]. PS is suitable to collect the bone particles with optimal size and low heat generation, thereby minimizing the possibility of thermal necrosis [25].

A feature of the use of PS is the significant amount of surviving osteoblasts and osteocytes in bone blocks removed by ultrasonic surgery, besides that, the clinical outcomes sometimes cannot be seem when compared to surgery with rotary instruments [25]. Additionally, the surgical approach is easier at the oral cavity compared to surgical drills that use straight hand pieces, due to the angulation and small size of the piezoelectric tips and hand piece [16]. It is also reported that intraoperative bleeding, as well as bleeding-related complications, are minimal in surgeries where surgical ultrasound is employed, due to the physical phenomenon of cavitation [8]. The preparation of bone blocks for their adaptation over the surgical site in onlay-type grafts can also be performed by PS. Usually, the preparation is easier and safer, however it is more time consuming [7].

The risk of complications with the inadvertent penetration of the mandibular canal or damage to adjacent teeth is virtually eliminated in ultrasonic surgery. In addition, conventional rotary instruments produce excessive heat during osteotomies and this may affect the viability of cells and lead to thermal necrosis [27]. On the other hand, the PS is characterized by cavitation effect, with abundant cooler solution, yielding a harmless thermal effect, and resulting in better biological responses [28].

\section{Use for Alveolar Osteogenic Distraction}

Alveolar osteogenic distraction is used to increase the alveolar ridge height in patients requiring therapy with dental 
implants that have insufficient bone to ensure a proper crown-implant ratio at rehabilitation [29].

Gonzalez-Garcia et al. (2008) conducted a study of 17 vertical alveolar distractions in the posterior region of the mandible, 7 in the right side and 10 in the left side. The results were compared between two approaches: conventional technique (11) and piezoelectric technique (6). After analyzing several criteria, the authors concluded that the use of piezoelectric osteotomy in osteogenic distraction in order to increase the alveolar ridge height prior to the installation of dental implants is easier for the surgeon and less prone to intraoperative complications compared with conventional osteotomy procedures. However, the results also suggested that the piezoelectric osteotomy increases the risk of postoperative complications and reduces the overall success rate of rehabilitation, since the gap left after the piezoelectric osteotomy is bigger than that of osteotomy performed with conventional instruments, in which the bone-cutting completion is performed with thin chisels [2].

\section{Use for Lateralization of the Inferior Alveolar Nerve}

Typically, the amount of bone available for rehabilitation with dental implants in the posterior mandible is reduced, due to bone remodeling occurred following tooth loss in the region together with the presence of the inferior alveolar nerve. This may preclude implants placement without an increase in bone dimension [30].

Among the therapeutic approaches for the treatment of severely atrophic jaws, there is the lateralization of the inferior alveolar nerve with simultaneous implant placement [31]. This technique only demands one surgical procedure, ensures bicortical stability of the implant and requires a total treatment time of approximately 6 months. Its disadvantage is the risk of post-surgical neurosensory changes, including irreversible nerve damage and significant functional consequences [32].

The release of the inferior alveolar nerve involves inserting instruments meticulously through the bone wall, usually with difficult access. The risk of accidental damage to the inferior alveolar nerve during osteotomy is minimized by the piezoelectric cut. In addition, the selective nature of PS with vibration at specific ultrasonic frequency for hard tissues contributes to eliminate common complications and sequelae from the use of conventional rotary instruments [33]. The use of piezoelectric surgery in these cases is very interesting, because it allows a safe osteotomy and easy access to release the nerve [34].

In a clinical study [32], 10 lateralization surgeries of the inferior alveolar nerve and simultaneous installation of dental implants were performed with the aid of piezoelectric devices, in 9 patients. Several tests were conducted to evaluate the postoperative neurosensory consequences. Immediately after surgery, all patients had some degree of neurosensory function decreasing, but at the end of the treatment period, all patients had resolution of their surgical sequelae without complications. The results suggested that surgical transposition of the inferior alveolar nerve with the aid of PS is safe, since all patients in the study had a recovery of their sensory functions in a minimum period (maximum time for recovery, in this study, was 3 months; 8 of the 10 cases having full recovery in 2 weeks). The authors also concluded that, despite the high risk of temporary or permanent nerve damage, the use of PS allowed performing flaps and smaller osteotomies, and reduced total surgical time. It also provided better surgical control over the preparation of the neurovascular bundle and all surgical steps were completed faster [32].

\section{Use for Alveolar Bone Crest Expansion}

In cases with sufficient bone height, but insufficient thickness, bone expansion may be indicated [35]. Split the alveolar crest has been used primarily in the maxilla where bone elasticity is higher [13]. Piezosurgery has shown good results in these situations and the bone can be separated with no trauma. The entire length of the osteotomy can be expanded by inserting osteotomes. This approach allows to achieve the required depth [30].

During bone splitting, there is an additional risk of unwanted fracture, especially in predominantly cortical bone, when the osteotomy is performed with conventional rotary drills and oscillating saws. The PS method decreases the risk of bone fracture, resulting in a more elastic bone following osteotomy with ultrasonic vibration, thus minimizing complications [30].

\section{DISCUSSION}

In oral surgery, PS was introduced to carry out maxillary sinus lifting surgeries more safely [20]. However, new indications continue to appear due to its great performance in cutting bone tissue. Its characteristic of soft tissue preservation transforms procedures that were usually critical into simple and perfectly executable [36].

The most common intra-operative complication with sinus lift surgery is perforation of the Schneiderian membrane $[3,7,19]$. The perforation rate reported in the literature in surgeries performed by conventional technique without using the piezoelectric device ranges between 14 and 56\% [20], with an average of $30 \%$ [19]. According to the literature, for surgeries in which PS was employed, this rate fell. Some authors work with numbers between 5\% [18] and 7\% [19]. These authors also concluded that in most cases these perforations occurred during membrane handling with hand tools, rather than during the use of ultrasound.

Still in sinus lift surgeries, occasionally, during the use of rotary instruments, there may be bleeding from the cut of branches of anastomosis of the lower arm of the posterior superior alveolar artery and infraorbital artery, more often in vertical osteotomies in the posterior region of maxilla. This artery is present in $100 \%$ of cases [37]. With the use of PS this risk is greatly diminished or even eliminated. The piezosurgical device promotes a clean surgical area as it keeps it free from bleeding during bone cutting, due to the effect of air-water cavitation of the ultrasonic device. This allows a better view of the surgical site [20]. The cooling solution by hydropneumatic pressure assists in the Schneiderian membrane release [12] which minimizes the risk of perforations.

The main disadvantage of the piezosurgery in the bone augmentation procedures and osteotomies is its difficulty 
against the cortical bone [11,33]. Common donor sites for bone grafting, such as chin, mandibular ramus and parietal bones, are composed primarily of cortical bone. Thus, the use of PS, in theory, would be difficult. Nevertheless, the piezosurgical device is considered the best instrument available for collecting autogenous grafts, even in cortical bone [36]. Ultrasonic vibrations improve the breaking of solid interfaces and facilitate the cleavage of the graft from the donor site. The collection of bone blocks is performed without the use of chisel and hammer. These instruments are known for the violent impact and the risk of unwanted bone fracture [16]. Moreover, ultrasonic vibration allows cortical bone splitting while preserving the surrounding soft tissues [33]. The use of ultrasonic tips is extremely safe and effective, preserving vital structures such as nerves and blood vessels [8].

The hypothesis of bone overheating by the vibration of the tips still remains [38]. However, many authors have shown that the overheating generated by the use of rotary instruments is larger and more harmful to cells, compared with the use of piezoelectricity. Histological findings showed less thermal necrosis of bone collected by PS when compared with other methods [6].

The fact that the piezosurgical bone cutting produces less heat revealed in microphotographic and histomorphometric studies a less pronounced inflammatory response. When comparing high and low-speed burs, chisels, pliers, gougeshaped bone chisel, and piezoelectric device for autogenous bone harvesting, there was not a predominance of dead cells in the PS group as in others. It was found in the inflammatory process a greater number of regulatory proteins such as (BMP)-4, (TGF)- $\beta 2$, tumour necrosis factor $\alpha$, and interleukin-1 $\beta$ and -10 . The neo-osteogenesis process was also better with a rapid increasing in (BMP)-4 and (TGF)- $\beta 2$ (bony repair inducers) and a minor amount of pro-inflammatory proteins [39].

For a histological study, cortical bone particles were collected by piezoelectric ultrasound and conventional drills. The bone particles were compared by using histomorphometric analyses that investigate bone morphology, cell viability and differentiation. This study showed that autogenous bone particles collected with PS contained more cells that differentiated into osteoblasts [10]. Still on the biological aspect, studies have stated that the use of ultrasound for osteotomies reduces damage to osteocytes and promotes greater survival of living bone cells during autogenous bone graft. Additionally, they stated that the piezoelectric surgical technique is more effective in stimulating osteogenesis around implants, promoting greater number of osteoblasts in the implant receptor sites and reducing local inflammatory precursors $[14,39]$.

The increased surgical time during surgical preparation and osteotomies is also considered a problem by some authors. In fact, the PS technique does not increase the total surgical time of the procedures, because the time spent to protect the soft tissues is minimized [20]. Furthermore, the number of instruments required to perform the osteotomies in many cases is reduced to only the ultrasonic hand piece. This leads to a reduction on the time spent with the exchange of instruments [2].

\section{CONCLUSION}

The piezosurgical device is safe and effective to work on bone tissues. Primarily in dental implantology, the use of this device makes the dentists' work easier and provides comfort to patients. The advantages of piezoelectric surgery are protection of soft tissues, better visualization of the surgical field, reduction of noise and vibration, reduction in stress and fear of the patient, reduction of bleeding, and increase in comfort for the surgeon's work. Its use is increasing as well as the application areas.

\section{CONFLICT OF INTEREST}

The authors confirm that this article content has no conflict of interest.

\section{ACKNOWLEDGEMENTS}

Declared none.

\section{REFERENCES}

[1] Vercellotti T. Technological characteristics and clinical indications of piezoelectric bone surgery. Minerva Stomatol 2004; 53: 207-14.

[2] Gonzalez-Garcia A, Diniz-Freitas M, Somoza-Martin M, GarciaGarcia A. Piezoelectric and conventional osteotomy in alveolar distraction osteogenesis in a series of 17 patients. Int $\mathrm{J}$ Oral Maxillofac Implants 2008; 23: 891-6.

[3] Robiony M, Polini F, Costa F, Vercellotti T, Politi M. Piezoelectric bone cutting in multipiece maxillary osteotomies. J Oral Maxillofac Surg 2004; 62: 759-61.

[4] Callister WD. Materials Science and Engineering: An Introduction. New York: Techbooks 2008.

[5] Leclercq P, Zenati C, Dohan DM. Ultrasonic bone cut part 2: Stateof-the-art specific clinical applications. J Oral Maxillofac Surg 2008; 66: 183-8.

[6] Leclercq P, Zenati C, Amr S, Dohan DM. Ultrasonic bone cut part 1: State-of-the-art technologies and common applications. J Oral Maxillofac Surg 2008; 66: 177-82.

[7] Pavlikova G, Foltan R, Horka M, Hanzelka T, Borunska H, Sedy J. Piezosurgery in oral and maxillofacial surgery. Int $\mathrm{J}$ Oral Maxillofac Surg 2011; 40: 451-7.

[8] Happa A. Use of a piezoelectric surgical device to harvest bone grafts from the mandibular ramus: report of 40 cases. Int $\mathrm{J}$ Periodontics Restorative Dent 2007; 27(3): 241-9.

[9] Crosetti E, Battiston B, Succo G. Piezosurgery in head and neck oncological and reconstructive surgery: personal experience on 127 cases. Acta Otorhinolaryngol Ital 2009; 29: 1-9.

[10] Chiriac G, Herten M, Schwarz F, Rothamel D, Becker J. Autogenous bone chips: influence of a new piezoelectric device (Piezosurgery) on chip morphology, cell viability and differentiation. J Clin Periodontol 2005; 32: 994-9.

[11] Stubinger S, Kuttenberger J, Filippi A, Sader R, Zeilhofer HF. Intraoral piezosurgery: preliminary results of a new technique. J Oral Maxillofac Surg 2005; 63: 1283-7.

[12] Vercellotti T, Nevins ML, Kim DM, et al. Osseous response following resective therapy with piezosurgery. Int J Periodontics Restorative Dent 2005; 25: 543-9.

[13] Schlee M, Steigmann M, Bratu E, Garg AK. Piezosurgery: basics and possibilities. Implants Dent 2006;15: 334-40.

[14] Preti G, Martinasso G, Peirone B, et al. Cytokines and growth factors involved in the osseointegration of oral titanium implants positioned using piezoelectric bone surgery versus a drill technique: a pilot study in minipigs. J Periodontol 2007; 78: 716-22.

[15] Robiony M, Polini F, Costa F, Toro C, Politi M. Ultrasound piezoelectric vibrations to perform osteotomies in rhinoplasty. J Oral Maxillofac Surg 2007; 65: 1035-8.

[16] Sohn DS, Ahn MR, Lee WH, Yeo DS, Lim SY. Piezoelectric osteotomy for intraoral harvesting of bone blocks. Int $\mathrm{J}$ Periodontics Restorative Dent 2007; 27: 127-31. 
[17] Schaeren S, Jaquiery C, Heberer M, Tolnay M, Vercellotti T, Martin I. Assessment of nerve damage using a novel ultrasonic device for bone cutting. J Oral Maxillofac Surg 2008; 66: 593-6.

[18] Vercellotti T, De Paoli S, Nevins M. The piezoelectric bony window osteotomy and sinus membrane elevation: introduction of a new technique for simplification of the sinus augmentation procedure. Int J Periodontics Restorative Dent 2001; 21: 561-7.

[19] Wallace SS, Mazor Z, Froum SJ, Cho SC, Tarnow DP. Schneiderian membrane perforation rate during sinus elevation using piezosurgery: clinical results of 100 consecutive cases. Int J Periodontics Restorative Dent 2007; 27: 413-9.

[20] Baldi D, Menini M, Pera F, Ravera G, Pera P. Sinus floor elevation using osteotomes or piezoelectric surgery. Int J Oral Maxillofac Surg 2011; 40: 497-503

[21] Eggers G, Klein J, Blank J, Hassfeld S. Piezosurgery: an ultrasound device for cutting bone and its use and limitations in maxillofacial surgery. Br J Oral Maxillofac Surg 2004; 42: 451-3.

[22] Flemmig TF, Petersilka GJ, Mehl A, Hickel R, Klaiber B. The effect of working parameters on root substance removal using a piezoelectric ultrasonic scaler in vitro. J Clin Periodontol 1998; 25: $158-63$.

[23] Sivolella S, Berengo M, Scarin M, Mella F, Martinelli F. Autogenous particulate bone collected with a piezo-electric surgical device and bone trap: a microbiological and histomorphometric study. Arch Oral Biol 2006; 51: 883-91.

[24] Misch CM. Comparison of intraoral donor sites for onlay grafting prior to implant placement. Int J Oral Maxillofac Implants 1997; 12: 767-76.

[25] Berengo M, Bacci C, Sartori M, Perini A, Della Barbera M, Valente M. Histomorphometric evaluation of bone grafts harvested by different methods. Minerva Stomatol 2006; 55: 189-98.

[26] Degerliyurt K, Akar V, Denizci S, Yucel E. Bone lid technique with piezosurgery to preserve inferior alveolar nerve. Oral Surg Oral Med Oral Pathol Oral Radiol Endod 2009; 108: e1-5.
[27] Schlee M. Piezosurgery a precise and safe new oral surgery technique. Aust Dent Pract 2009; 2009: 144-8.

[28] Garcia-Garcia A, Somoza-Martin M, Gandara-Vila P, Saulacic N, Gandara-Ray JM. Alveolar distraction before insertion of dental implants in the posterior mandible. Br J Oral Max Surg 2003; 41: 376-9.

[29] Atwood DA. Bone loss of edentulous alveolar ridges. J Periodontol 1979; 50: 11-21

[30] Jensen O, Nock D. Inferior alveolar nerve repositioning in conjunction with placement of osseointegrated implants: a case report. Oral Surg Oral Med Oral Pathol 1987; 63: 263-8.

[31] Bovi M. Mobilization of the inferior alveolar nerve with simultaneous implant insertion: a new technique. Case report Int J Periodontics Restorative Dent 2005; 25: 375-83

[32] Vercellotti T. Piezoelectric surgery in implantology: a case reporta new piezoelectric ridge expansion technique. Int J Periodontics Restorative Dent 2000; 20: 358-65.

[33] Schlee M. Piezosurgery: a precise and safe new oral surgery technique. Aust Dent Pract 2009; 38-142.

[34] Palti A. Ridge splitting and implant techniques for the anterior maxilla. [Interview]. Dent Implantol Update 2003; 14: 25-32.

[35] Pereira CC, Gealth WC, Meorin-Nogueira L, Garcia-Júnior IR, Okamoto R. Piezosurgery applied to implant dentistry: clinical and biological aspects. J Oral Implantol 2012 [Epub Ahead of Print].

[36] Solar P, Geyerhofer U, Traxler H, Windisch A, Ulm C, Watzek G. Blood supply to the maxillary sinus relevant to sinus floor elevation procedures. Clin Oral Impl Res 1999; 10: 34-44.

[37] Happe A. Use of a piezoelectric surgical device to harvest bone grafts from the mandibular ramus: report of 40 cases. Int Periodontics Restorative Dent 2007; 27: 241-9.

[38] Müller CK RG, Nentwig GH. Microscopical examination of osteotomies with ultrasonic, laser and classical procedures [abstract]. Clin Oral Impl Res 2007; 18: LXIII.

[39] Labanca M, Azzola F, Vinci R, Rodella LF. Piezoelectric surgery: twenty years of use. Br J Oral Maxillofac Surg 2008; 46: 265-9.

(C) Magrin et al.; Licensee Bentham Open.

This is an open access article licensed under the terms of the (https://creativecommons.org/licenses/by/4.0/legalcode), which permits unrestricted, noncommercial use, distribution and reproduction in any medium, provided the work is properly cited. 